\title{
Editorial special issue: house, home and dwelling
}

\author{
Henny Coolen $\cdot$ Janine Meesters
}

Published online: 4 October 2011

(c) The Author(s) 2011. This article is published with open access at Springerlink.com

\begin{abstract}
The aim of this special issue is to further our understanding of 'house', 'home' and 'dwelling' by presenting five empirical studies that investigate different aspects of these concepts. All three are complex, multi-faceted and multi-layered concepts, whose diverse connotations are often used interchangeably. For example, the word home is used for the physical structure of the house, for the meanings attached to the house, as well as for the process of homemaking. From an analytical point of view this is undesirable, since we require our concepts to be as unambiguous as possible. Therefore, in this introductory paper, the guest editors present a conceptual framework for studying house, home and dwelling that is based on the fundamental distinction between an environmental object and the affordances attached to it. The studies presented in this special issue investigate different aspects of house, home and dwelling, but they all use this conceptual framework and share the same theoretical perspective on people-dwelling relations. And, although each of the papers has its own merits, together they demonstrate that the conceptual framework is an effective tool for dismantling the concepts of house, home and dwelling.
\end{abstract}

Keywords House $\cdot$ Home $\cdot$ Dwelling $\cdot$ Affordance $\cdot$ Meaning

\section{Introduction}

The concept of home and the ideas, interpretations and meanings associated with it have been constantly present in the academic literature over the last two decades (Després 1991; Benjamin and Stea 1995; Moore 2000; Mallett 2004; Blunt and Dowling 2006). What all these publications have in common, despite their differences in approach and focus, is that they acknowledge that 'home' is a complex, multi-faceted and multi-layered concept,

H. Coolen $(\bowtie)$

OTB Research Institute for the Built Environment, Delft University of Technology, Delft,

The Netherlands

e-mail: h.c.c.h.coolen@tudelft.nl

J. Meesters

Municipality of 's-Hertogenbosch, 's-Hertogenbosch, The Netherlands 
whose different connotations are often used interchangeably and/or simultaneously. Although some authors (Blunt and Dowling 2006) do not seem to find this disturbing, it has led others (Rapoport 1995) to plead for abandoning the concept altogether. This has some appeal from an analytical point of view, since we require our concepts to be as clear and unambiguous as possible. On the other hand, one might argue that the word home is used both in the academic literature and in daily speech and that we should accept this as a fact of life (cf. Lawrence 1995). Interestingly, not all languages and cultures seem to have a word for home (Benjamin 1995). So if one wants to use it, it is the researcher's task to demarcate the concept to clarify in which facets one is interested (cf. Mallett 2004). This may be done in a formal way by using methodologies such as the facet approach (Canter 1983) that aim at dismantling complex concepts or, in a less formal way, by just indicating which connotations one emphasizes. Both approaches are taken in this special issue.

This special issue contains five empirical studies on people-dwelling relations. Even though each one investigates a different aspect of house, home and dwelling, they all are based on the same conceptual framework and share the same theoretical perspective. In this editorial we present this conceptual framework and briefly introduce each contribution to this special issue on House, Home and Dwelling.

\section{House, home and dwelling}

\subsection{House and dwelling}

In housing research, the word commonly used to describe the physical structures we live in is house. Given the fact that housing research is mainly Western oriented, this term seems to be synonymous with the Western type of single-family house. Oliver (2003) notes that $90 \%$ of the dwellings in the world are not of this type but are what he calls vernacular dwellings, which are buildings that are self-built by their owner-occupiers or built by members of the community in which the occupier lives. Sometimes we recognize that not all dwellings are houses, for instance when certain types of dwellings are characterized as slums, huts, cabins, shacks or tents. So the term house seems to indicate a certain Westernoriented type of dwelling, which, from a cross-cultural point of view, makes it less useful. Recognizing this, Rapoport (1980) has applied the generic term dwelling to all physical structures used by people for living, and we follow his lead.

A dwelling is defined as the system of settings, being a subsystem of the environment, that affords certain systems of functions which make it for the inhabitants the primary anchor in the environment (Rapoport 1990). These systems of functions comprise activity systems, such as eating, sleeping, relaxing, and entertaining family and friends, but may also include socio-psychological functions, for example family life, safety, and privacy. The term primary anchor indicates that a dwelling is the operating base that provides shelter and concealment, and the place from which most people undertake activities, explore and experience the world and where they return.

Considering a dwelling as a subsystem of the environment makes it possible to understand its specific functions, such as a place of retreat, not only in terms of its occupiers but also in the context of the other subsystems in the environment. Only a subset of all the relevant functions is afforded by a dwelling. This subset may be different for different individuals and the subsystem of settings that makes up the dwelling may also vary. It may include, for instance, a certain number of rooms, a garden, a driveway, a garage, a certain layout, an attic, and many other settings. This does not mean that a 
dwelling is completely idiosyncratic and that there are no common or shared conceptions of what a dwelling is in terms of settings and functions. As several authors (Rapoport 1969; Oliver 2003) have argued, a dwelling can be considered as a cultural artefact, which implies the existence of more or less shared conceptions of dwellings within cultures.

The environment may be conceived as consisting of several subsystems, one of which forms the dwelling. Another important subsystem is the dwelling environment, which consists of those settings that afford functions which the inhabitants want to be realized in the immediate vicinity. The dwelling environment may include shops, a school, a park, a church, a playground, and many other settings.

Given the above definitions of the dwelling and the dwelling environment, it is evident that dwelling, as a verb, comprises more than activities; it encompasses the performance and experience of all the affordances that make it a dwelling for the individual.

\subsection{Home}

The concept of home and its ideas, interpretations and meanings have attracted quite some attention in the academic literature in the last two decades (Després 1991; Benjamin and Stea 1995; Moore 2000; Mallett 2004; Blunt and Dowling 2006). Given the way it is applied in this literature, there are at least five facets of home that can be deduced and that may aid in dismantling the concept. Before describing these facets, it is worth noting a bias towards using the concept mainly in positive terms (Rapoport 1995; Blunt and Dowling 2006). Home seems to be embedded in positive feelings and affective bonds; negative feelings, for instance those associated with broken families or domestic violence, have received little attention, with few exceptions (Anthony 1997; Gram-Hanssen and BechDanielsen 2008).

The first facet that can be deduced entangles an environmental object with the relationships people may have with this object. In terms of housing, the concept of home is used for the material structure (Després 1991), the physical aspects (Moore 2000), and the place or site (Blunt and Dowling 2006) in which we live, as well as the meanings we attach to the physical structure (Rapoport 1995; Moore 2000), which are characterized by Blunt and Dowling (2006) as 'the experience and meaning of home' or 'home as an idea and an imaginary imbued with feelings'. According to Rapoport (1995), the original meanings of dwelling and home were in terms of this facet. House and dwelling were used to indicate the physical structure, while home was used for the relationships we experience with the physical structure and the meanings we attach to it. Given this distinction the use of the phrase meaning of home seems tautological, and a sign saying home for sale is meaningless. The articles in this special issue use dwelling or house for the physical structure and home for the meaning attached to this physical structure, or certain aspects of it.

The second facet that appears in the literature on home is the spatial dimension. The term home is being used for such different spatial entities as house, neighbourhood, town, state, and country (Mallett 2004; Blunt and Dowling 2006), for instance in the words hometown, home state and homeland. Sometimes the term home is used to denote several of these different spatial connotations simultaneously. For instance, a person who is currently in a foreign country and tells us that he is going home tomorrow probably means that he is returning to his homeland but also to his dwelling. Although these different spatial connotations of home occur in the literature, there is a strong emphasis on home in the sense of the dwelling (Moore 2000; Blunt and Dowling 2006). All the papers in this special issue address one or more aspects of this spatial facet. For instance, Reuschke investigates the meaning of dwelling in different locations; Coolen and Meesters focus on the meaning 
of two particular settings, namely the private garden and public green space; and Buckenberger investigates the meaning of a type of dwelling environment, the suburb.

The temporal facet is the third dimension that appears in studies about home. In many studies about home the focus is the current dwelling and, in general, the current time period. But sometimes, when speaking of home, one is referring to the place where one was born and/or raised or had once happily lived, for instance after a divorce (Anthony 1997). In this context Brink (1995) distinguishes the real home, the place where one was born, from the present home, the place where one currently lives. The temporal facet may also be focused on the future. This could occur when individuals do not consider the place where they were born and raised as a home-for instance because of broken families or domestic violence - and express that they would someday like to have a home for themselves and/or their children. The temporal facet of home plays a role most clearly in the paper by GramHanssen and Bech-Danielsen.

The fourth facet refers to social relations. Blunt and Dowling (2006) remark that home is often considered from the perspective of the family or the household. This seems to be a matter of course, as we have already noted that the emphasis is often on the home connotation of dwelling. But other groups—such as a group of friends, in the case of a commune; a group of monks or nuns, in the case of a monastery; a barrack in the case of the army; or an ethnic group, for instance in the expression 'I am going home to my people'-may also carry the connotation of home. Home in the sense of social relations appears most clearly in the paper by Bélanger.

A fifth facet relates to the distinction between product and process. In many of the meanings of the concept that we have described so far, home is considered as a place and/ or in terms of people's relationships with a place. However, the term home is also used for the process of homemaking (Rapoport 1995) and sometimes even as the process of establishing one's identity in society (Blunt and Dowling 2006).

There still seem to be other connotations of the term home that are not directly related to one of these facets. For instance, home as the place where something is invented, founded or developed as in the expression the US is the home of baseball (Mallett 2004) or home in the sense of being familiar or conversant with, as in such expressions as the truck driver is at home on the highway or the working woman is at home in the spinning mill (Heidegger 1971). These meanings will not be dealt with here.

\section{Conceptual framework}

An individual's operating environment consists of objects, the things toward which the individual is oriented; they form the focal points around which the individual's activities become organized. An object is anything that can be referred to or designated; objects may be material or immaterial, real or imaginary, in the outer world or inside the body, have the character of an enduring substance or be a passing event. From the perspective of a human being, the environment may be classified in at least five categories: other human beings, other animals, physical objects, social objects, and abstract objects. If the individual notes or is aware of any one of these things, it is an object for that individual. Objects constitute the world or operating environment of the human being. Taken together, they constitute the individual's world of existence, that is, the things the individual deals with in life activity.

Objects have meaning for human beings in terms of the possibilities they offer for actions and intentions; that is, an object may have certain features in relation to a goal of the individual. The concept of affordances (Gibson 1986) most basically highlights this 
congruence between structural features of the environment and the intentions and goals of individuals. Affordances are relations between features of objects and abilities of human beings (Chemero 2003); they are attributable to the intrinsic features that objects possess by virtue of their make-up, and are specified in relation to the individual. For example, a firm, obstacle-free ground surface affords walking on, a chair affords sitting on, a door to a room affords opening and passage, a dwelling affords shelter, a room affords privacy, a certain form of tenure affords independence. So affordances are mutual relationships that point both at environmental features and at human beings. At a functional level of analysis environmental features are experienced in terms of their affordances, i.e. their meaning, for the individual.

The features of the environment are only one facet of a dynamic individual-environment relation; the other facet is intentional actions of individuals, and this aspect of the individual—environment relation becomes most apparent in the selection, the discovery, and the creation of meaningful environmental features (Heft 2001). Individuals selectively engage particular objects in their surroundings; individuals typically make choices from among the range of potential features in a setting to support some activity. However, individuals do not have unconstrained choice. Factors outside their control may limit the range of socially and/or culturally sanctioned choices. So there is self-selection of affordances but often within constraints.

Intentionality is also apparent in the processes through which individuals learn about and discover the features of objects and the affordances in their surroundings. This is not a random process; which objects are selected in the first place is delimited by the perceived congruence between an object's features and the individual's functional capabilities and intentions. This reciprocity gives rise to exploration and discovery within constraints. Finding novel uses for familiar objects is a particularly satisfying way to discover new affordances.

Actions involving learning about environmental features are frequently guided by others. Throughout life, most apparently during childhood, individuals are explicitly taught, often in very subtle ways, to recognize and utilize the functional features of objects. Individuals also learn about the meanings of objects by observing the actions of others.

Finally, affordances are sometimes created when the range of possibilities available in the environment are insufficient to meet certain goals. The environment is comprised of meaningful features that were created by an individual or a group of individuals at some time. This omnipresent fact about the world is one manifestation of the fundamental reciprocity of individuals and their environment. Individuals do not merely take the world as they find it; the environment is continually being modified. Many of these activities are efforts to create new affordances in order to address specific individual and socio-cultural needs.

This ubiquity of affordances points to an important issue. In many cases, meaningful features of the environment that are created reflect an individual's knowledge about environment-behaviour relations. This means that a great deal of what is known is embodied in the environmental structures individuals create; we live our lives in environments rich in what might be called ecological knowledge. In this functional sense every object has a meaning that distinguishes it from other objects. This meaning constitutes the nature of the object for the individual for whom the object exists. One confronts an object, sees it, refers to it, talks about it, or acts toward it in terms of the meaning it has for one. No objects exist for a person except in terms of the meaning it has for the person. Meaning is not something that is inherent in an object; it is not an intrinsic part or attribute of the object. The meaning of an object exists in a relation between the object and the individual 
for whom it is an object; its meaning consists of how the individual designates the object, and in this sense an object may have different meaning for different human beings.

The pair environmental object-affordance forms the basis of the conceptual framework presented in Fig. 1 (Coolen 2006). This framework shows the interrelations among the individual, affordances and environmental objects. At the same time it depicts how these phenomena and interrelations are embedded in the social, cultural, economic and political system. Environmental objects potentially have many and diverse affordances. Whenever a function is assigned to a feature a relationship arises between the feature and the function, which is called an affordance (cf. Chemero 2003). This relationship originates from the individual that assigns the function. It is relative to the individual in the sense that the relationship between a function and a feature may be possible for some individuals but not for others. The dotted line in Fig. 1 indicates this relativity of the individual-environment relationship. For instance, a chair may afford sitting on for some individuals but not for others due to their size. So the term affordance is reserved here for the direct relation between a feature and a function that is assigned to it by an individual, whatever the nature of that function may be. In this sense affordances may be considered as basic meanings (cf. Chemero 2003).

The relativity of the individual-environment relationship, which has so far been illustrated in terms of abilities or attitudes, is also relevant in another sense (Coolen 2008). This concerns, for instance, socio-demographic variables such as income, age and household composition. These variables condition individual-environment relations in the sense that they determine to a certain extent whether potential affordances may become actual affordances. For instance, a certain dwelling may potentially afford all the affordances one is looking for, but these affordances may not materialize because one cannot afford the dwelling financially. And a certain dwelling may afford a separate room for every family member in the case of some families but not others due to the size of the household.

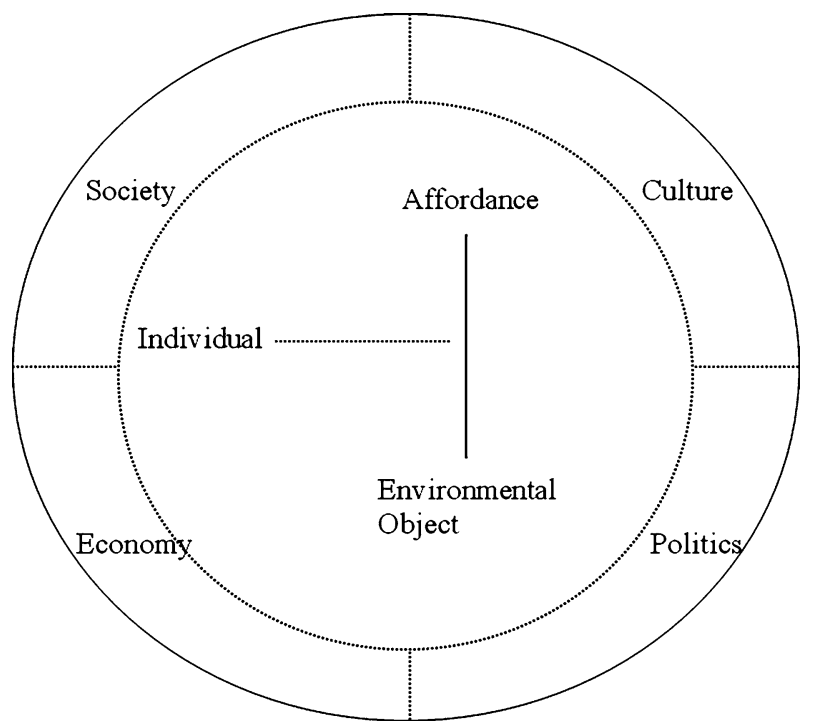

Fig. 1 Conceptual framework for studying people-environment relations 
In the context of this special issue about house, home and dwelling a few remarks about the environmental object and its affordances would seem worthwhile. The environmental object could be the dwelling that, seen holistically, affords such functions as shelter, sleeping, eating, entertaining guests, security and concealment. It could be the dwelling layout that may or may not be adequate to afford all the functions the inhabitants want. The focus can be on dwelling features such as the garden that may afford gardening and relaxation, or the number of rooms that may afford privacy for all inhabitants of the dwelling. The environmental object can be a setting of the dwelling environment such as a park that may afford being outside or meeting people, but it can also be the dwelling environment, seen holistically, that may or may not afford safety. The affordances of the settings are the meanings which are denoted above by the term home. This implies that by clearly specifying the environmental object one is considering and by indicating the associated timeframe it becomes immediately clear which facet of home-spatial, temporal, social relations, product or process-one is considering.

Enumerating the settings of the dwelling and/or dwelling environment and the associated affordances results in a quality profile of the dwelling or dwelling environment (cf. Rapoport 2005). The positive or negative evaluation of each element in such a profile indicates one's satisfaction with this quality aspect. At the same time each quality-aspect evaluation contributes to one's overall satisfaction with the dwelling and/or dwelling environment.

The description of the conceptual framework so far has mainly emphasized the interrelations between the individual, an environmental object and an affordance. These aspects are, as indicated above, only one facet of a dynamic individual-environment relation; another facet consists of the intentional actions of individuals, which become most apparent in the selection, the discovery, the learning, and the creation of meaningful environmental features. These processes have been sketched above as taking place in society at large, which implies that they are shaped, influenced and constrained by social, cultural, economic and political factors, and that they in turn have an impact on these factors. These facets of the conceptual framework are not treated further here. To do so would require specifying what these factors exactly are and elaborating how these processes really work in the making of environmental features and their meanings. Yet they are addressed in several of the papers in this special issue.

\section{Contents of the special issue}

The first paper focuses on the locational dimension of home. In particular it deals with aspects of multi-locational dwelling for job-related reasons. Based on both quantitative and qualitative data, Reuschke investigates dwelling conditions, preferences, experiences and meanings in a job-induced way of living with two accommodations. Reuschke found five different types of commuter dwelling arrangements, which are based on objective dwelling conditions and subjective dwelling preferences. These vary from commuters who can realize their preferences for higher amenity values to commuters who cannot fulfil their dwelling preferences at the secondary residence. The meanings attached to the secondary dwelling may also differ. For some the secondary dwelling is merely a working base that provides shelter, for others it is also a place of well-being and intimacy. The dwelling conditions and preferences at the job-used secondary residence are influenced by the particular commuting arrangement, household/family lifecycle and employment conditions. 
The second contribution to this special issue is about the effect of neighbourhood transformation on people's definition of their home territory. Bélanger mapped patterns of people's everyday activities in the Southwest Borough in Montreal (Canada), which is a former industrial area with a disused canal and many affordable houses. When the canal was reopened with a park along it, the area also became attractive to wealthier households. After 30 years of demographic and economic decline, the Southwest Borough is now witnessing growth again. While the long-term residents feel pushed away by the development of more luxurious buildings and shops, the new residents have appropriated the park. Although the long-term residents still use the park, they no longer see it as part of their home territory.

Coolen and Meesters extend the conceptual framework presented in the previous section by explicitly incorporating meanings in the model to investigate whether public green space may be a substitute for the private domestic garden. There seems to be a gap between consumer preferences and urban planning practice as far as outdoor space is concerned, at least in the Netherlands. Consumers have an increasing demand for spacious and green living, while governments promote the compact city from a sustainability point of view. In the course of urban development, the latter approach often results in the substitution of public green space for the private domestic garden. However, a representative survey among Dutch households shows that both settings have more or less unique affordances and meanings. People consider the domestic garden as an outdoor extension of the dwelling that affords casual leisure. Public green space is mainly valued for its contribution to the liveability of the dwelling environment and to the experience of nature. These key features imply that the private domestic garden cannot simply be substituted by public green space in the Netherlands.

Buckenberger investigates the affordances of housing quality from a resident's point of view. She interviewed people living in the suburb of Farm Cove in Manuka City in the Auckland region, New Zealand. There are two predominant age groups in this area: the long-term residents who moved in around 1970 during the period of suburbanization; and families in their thirties and early forties who moved in recently. Both groups attach different affordances to housing quality. The younger group emphasizes physical attributes when talking about housing quality (large, outdoor space, school, quiet), while the older people put more emphasis on intangible qualities (good life, neighbourhood, neighbours). So, living for a long period in a certain place seems to diminish the value placed on physical attributes, while the importance of intangible or even emotional qualities increases.

The final paper is about dwelling and home among immigrants in Denmark. It investigates whether the dwelling has a special significance for Somali, Iraqi and Turkish immigrants - home as a product - as well as the ways in which groups with different cultural roots establish their home-home as a process - in Danish social housing. The cultural background of immigrants does to some extent influence their housing needs. For example, the Danish floor plan does not always fit the housing needs of immigrant families. Having a separate kitchen that can be closed off is not always afforded by Danish social housing. On the other hand, the analysis suggests that the dwelling is as important for immigrants as for other residents and that immigrants often attach the same affordances to the dwelling.

\section{Conclusion and common themes}

This special issue contains five empirical studies on people-dwelling relations. They share to a large extent the same theoretical perspective on people-dwelling relations and 
use the conceptual framework presented in this editorial. The primary goal of this conceptual framework is to offer the researcher a tool to dismantle the complex concepts of house, home and dwelling by making the basic distinction between an environmental object and the affordances that are attached to this object by its users. All the papers clearly show that the framework fulfils this goal well and that not only the researchers but also the studied populations are able to distinguish the environmental object-be it the dwelling, the neighbourhood, the suburb or green space-from its affordances. The affordances that appear in the different papers may be characterized as being of two types. On the one hand, manifest affordances such as eating, sleeping, shopping, being outside, leisure activities and shelter appear in the studies. On the other hand, more latent affordances such as wellbeing, sense of community, privacy, peace and quiet and sense of security are attached to the studied environmental objects. This distinction between manifest and latent affordances is very similar to the distinction between manifest and latent functions made by Rapoport (1988). Overall we may conclude that the conceptual framework provides a simple but effective model for a more systematic study of house, home and dwelling.

Although the papers presented in this special issue investigate different aspects of house, home and dwelling, several papers also share some common themes. An important aspect of two papers-one by Bélanger and the other by Gram-Hanssen and Bech-Danielson-is their focus on adapting to a new environmental object and its consequences for the affordances attached to this new object. Bélanger investigates how indigenous residents adapt to both physical and social changes in their neighbourhood, and how these changes affect the affordances attached to the park in the neighbourhood. Gram-Hanssen and BechDanielson study how people who migrated to another country adapt to their new dwelling and how the process of creating a home develops.

A common aspect of another two papers-one by Reuschke and the other by Coolen and Meesters - is that they both concern the affordances of differently located but otherwise similar environmental objects. Reuschke studies the similarities and differences in affordances attached to the primary and job-induced secondary dwelling. Coolen and Meesters compare the affordances and meanings of two environmental objects- the private domestic garden and public green space-in the dwelling environment.

Finally, several papers consider whether the phases in the life course affect the affordances of certain environmental objects. Buckenberger compares the affordances attached to the dwelling and dwelling environment by young versus elderly couples. Reuschke studies the relationship between household composition and the affordances attached to the primary and secondary dwelling. And Coolen and Meesters investigate how the affordances of the private garden and public green space are affected by household composition.

Open Access This article is distributed under the terms of the Creative Commons Attribution Noncommercial License which permits any noncommercial use, distribution, and reproduction in any medium, provided the original author(s) and source are credited.

\section{References}

Anthony, K. H. (1997). Bitter homes and gardens: The meanings of home to families of divorce. Journal of Architectural and Planning Research, 14, 1-19.

Benjamin, D. N. (1995). Afterword, or further research issues in confronting the home concept. In Benjamin and Stea. pp. 293-309.

Benjamin, D. N., \& Stea, D. (1995). The home: Words, interpretations, meanings, and environments. Aldershot: Avebury. 
Blunt, A., \& Dowling, R. (2006). Home. London: Routledge.

Brink, S. (1995). Home: The term and the concept from a linguistic and settlement-historical viewpoint. In Benjamin and Stea. pp. 17-25.

Canter, D. (1983). The purposive evaluation of places: A facet approach. Environment and Behavior, 15, $659-698$.

Chemero, A. (2003). An outline of a theory of affordances. Ecological Psychology, 15, 181-195.

Coolen, H. (2006). The meaning of dwellings: An ecological perspective. Housing, Theory and Society, 23, $185-201$.

Coolen, H. (2008). The meaning of dwelling features: Conceptual and methodological issues. Amsterdam: IOS Press.

Després, C. (1991). The meaning of home: Literature review and directions for future research and theoretical development. Journal of Architectural and Planning Research, 8, 96-115.

Gibson, J. J. (1986). The ecological approach to visual perception. Mahwah: Erlbaum.

Gram-Hanssen, K., \& Bech-Danielsen, C. (2008). Home dissolution: What happens after separation? Housing Studies, 23, 507-522.

Heft, H. (2001). Ecological psychology in context. James Gibson, Roger Barker, and the legacy of William James' radical empiricism. Mahwah: Erlbaum.

Heidegger, M. (1971). Building, dwelling, thinking, in: Poetry, language, thought. New York: Harper \& Row.

Lawrence, R. J. (1995). Deciphering home: an integrative historical perspective. In: Benjamin and Stea. pp. 53-69.

Mallett, S. (2004). Understanding home: A critical review of the literature. The Sociological Review, 52, 62-89.

Moore, J. (2000). Placing home in context. Journal of Environmental Psychology, 20, 207-217.

Oliver, P. (2003). Dwellings. The vernacular house world wide. London: Phaidon.

Rapoport, A. (1969). House, form and culture. Englewood Cliffs: Prentice Hall.

Rapoport, A. (1980). Towards a cross-culturally valid definition of housing. In R. R. Stough \& A. Wandersman (Eds.), Optimizing environments-research, practice and policy, EDRA 11. Washington: EDRA.

Rapoport, A. (1988). Levels of meaning in the built environment. In F. Poyatos (Ed.), Cross-cultural perspectives in nonverbal communication (pp. 317-336). Toronto: C.J. Hogrefe.

Rapoport, A. (1990). Systems of activities and systems of settings. In S. Kent (Ed.), Domestic architecture and the use of space (pp. 9-20). Cambridge: Cambridge University Press.

Rapoport, A. (1995). A critical look at the concept home. In Benjamin and Stea. pp. 25-53.

Rapoport, A. (2005). Culture, architecture, and design. Chicago: Locke Science. 